\title{
XVI.
}

\section{Zwei Fälle von Endothelioma tuberosum colloides (Lymphangioma tuberosum multiplex Kaposi) nebst einigen Bemerkungen über die Lymphgefässe der Cutis.}

\author{
Von Dr. Ernst Kromayer, \\ Privatdocenten der Dermatologie in Halle a, $\mathbf{s}$.
}

(Hierzu Taf. IX. Fig. 1-2.)

I. Frau X., 32 Jahre alt. Der obere Theil der Brust, Hals und im geringen Maasse der untere Theil des Gesichtes mit zahlreichen mohn- bis linsengrossen Knötchen besetzt, die theils in Gruppen und Reihen angeordnet, theils nnregelmässig vertheilt sind. Die derben Knötchen liegen in der Cutis und sind nur mit ihr verschieblich. Die Farbe gelblich bis gelblichbraun. An einzelnen Knötchen transparente submiliare Krhabenheiten, die serösen Bläschen ähneln. Beim Einstich entleert sich indessen keine Flüssigkeit. Spaltet man über diesen bläschenartigen Erhabenheiten die bedeckende Haut, so lässt sich ein winziges, ziemlich derbes, transparent gelbliches Kügelchen herausdrücken. Die Affection besteht angeblich seit 6 Jahren und hat sich in letzter Zeit auf das Gesicht ausgedehnt. Die mikroskopische Untersuchung eines excidirten Knötchens ergab folgenden Befund. Die Parenchymhaut (Epidermis und die Cutis vasculosa) ist völlig normal. Die Cutis propria oder reticularis von sehr zahlreichen unregelmässig dicken, theilweise zackigen Zellsträngen grösstentheils in der Ricbtung der Langer'schen Spaltlinien durchsetzt, die vielfach an einer oder mehreren Stellen eystische Ausbuchtungen aufweisen, welche durch ibre Anzahl und Grösse dem Bilde etwas Charakteristisches verleihen (Tafel IX, Fig. 1). Das subcutane Gewebe frei von Veränderungen. Betrachten wir die Cutis propria in der Geschwulst etwas genauer, so füllt uns zunäehst der grosse Zellreichthum gegenüber der normalen (mitexcidirten) Haut auf. Die Zellkerne sind vielfach grösser, bläschenförmig nnd liegen bäufig zu zweien, dreien und mebreren dicht neben einander oder stehen mit ibren Protoplasmaausläufern in sichtbarer (Hämatoxylinfärbung, Glycerinmontirung) Verbindung, so dass kleine schmale Zellreihen entstehen (Fig 1, a, b, c). Von diesen schmalen Zellreihen zu den erwähnten Zellsträngen (Fig. 1, d, e,f, g) finden sich alle Uebergänge. Die cystischen Ausbuchtungen, die auf dem Querschnitte als runde, ovale oder längliche Figuren erscheinen, zeigen durchgehends bei schwacher Vergrösserung jene transparente Beschaffen- 


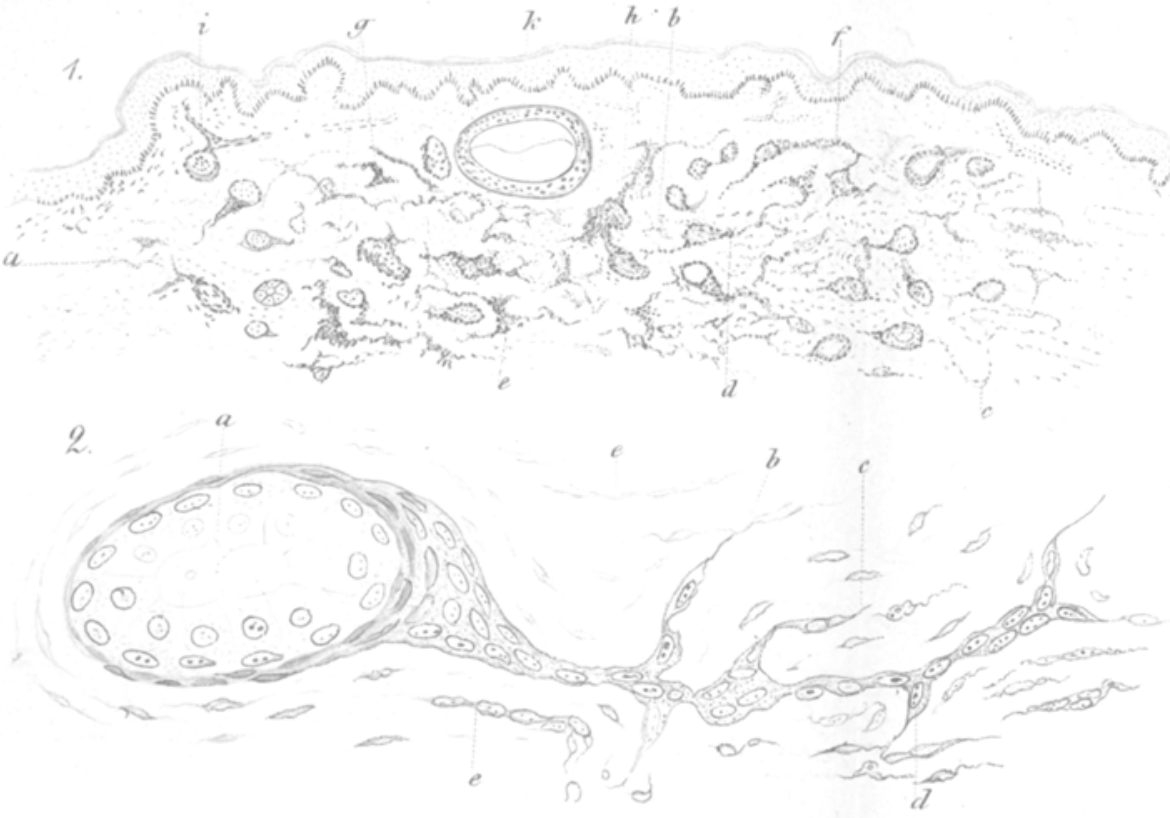

3.

L.

Zs

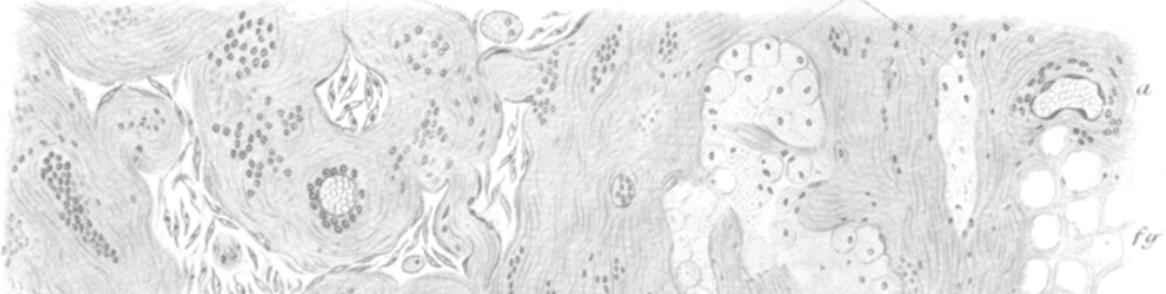

\section{(3) की}

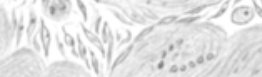

se $(4)$

$\mathrm{ig}^{\circ}, \mathrm{in}, \mathrm{m}$

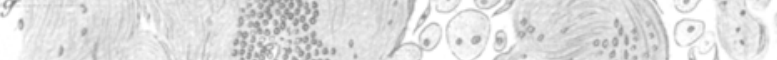

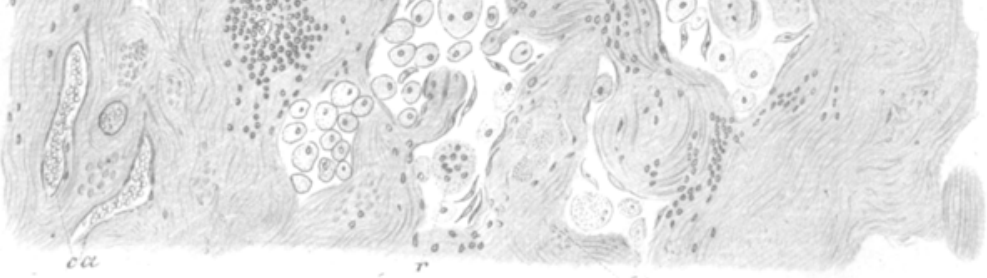

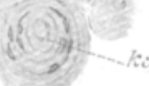

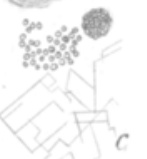

$2 \sqrt{6}$

\%

Winolimant si 
heit, die durch Behandlung der Schnitte mit Hämatoxylin, Carmin und Salzsäure als von colloider Substanz herrührend erkannt wird. Bei starker Vergrösserung kann man an geeigneten Stellen die Entstehuug der colloiden Substanz aus dem Protoplasma der Zellen verfolgen, deren Kerne und theilweise Zellcontouren sich noeb erhalten haben (Fig. 2, a). Die Grösse der Colloidcysten wechselt sehr, die grössten sind, wie vorber beschrieben, schon makroskopisch sichtbar. Die Zellschläuche sind, wie ihre Ausbuchtungen, stets solide. Nie babe ich ein Lumen an ibnen wahrgenommen; vielmebr liegen die Zellen dicht auf und neben einander, so dass sie an einzelnen $A$ bschnitten den Eindruck von Riesenzellen machen. Dabei bestehen (wie das Fig. 2, b, c, d zeigt) zwischen den die Zellschläuche zusammensetzenden Zellen und denen des umgebenden Outisgewebes directe protoplasmatische Verbindungen. Etwas wie eine Membrana limitans habe ich weder an den Zellstrăngen, noch den cystischen Ausbuehtungen beobachtet.

Dem Gesagten zu Folge haben wir eine Wucherung der festen Bindegewebszellen der Cutis vor uns, die ohne die geringste Zerstörung des Cutisgewebes sich zwischen den collagenen Bündeln, also nach unseren jetzigen Vorstellungen in den Bindegewebs- oder Lymphspalten ausbreitet: ein Endotheliom, dessen bindegewebige Gerüstsubstanz das Cutisgewebe selbst bildet. In der Literatur sind nur wenige Fälle vorhanden, die nach makround mikroskopischer Beschreibung wahrscheinlich zu diesem zu rechnen sind. Sie sind von den betreffenden Autoren mit folgenden Namen belegt worden: Lymphangioma tuberosum multiplex (Hebra-Kaposi) ${ }^{1}$ ), (Lesser-Beneke) ${ }^{2}$ ), Haemangioendothelioma tnberosum multiplex $(\mathrm{J} \text { arisch })^{3}$ ), Hydradenomes eruptives (Jaquet und Darier $)^{4}$ ), Syringocystadenoma (Török) ${ }^{5}$ ), Adenoma of the sweat glands (Perry $)^{6}$ ), Cystadenomes épitheliaux benins $(\text { Besnier })^{7}$ ), Cellulome épitheliales eruptif $(\text { Quinquaud) })^{8}$.

Bezüglich der Identität dieser Geschwülste unter einander

1) Haudbuch von Hebra-Kaposi. Bd.II. S. 292.

3) Dieses Archiv. Bd. 123.

3) Jarisch, Archiv für Derm. 1894. Bd. 28. S. 163.

4) Annales de Dermat. 1887. Bd. VIII.

5) Monatsh. f. prakt. Derm. 1889. Bd. VIII.

6) Interuationaler Atlas seltener Hautkrankheiten. 1890.

${ }^{7}$ ) Pathologie et traitement des maladies de la Peau par Kaposi, traduction par Besnier et Doyen. 189.1. tome II. p. 367.

$\left.{ }^{8}\right)$ Congrès international de Dermatologie. Comptes rendus. 1889. 
verweise ich auf die Abhandlung von Philippson ${ }^{1}$ ), Jarisch ${ }^{2}$ ) und Török ${ }^{3}$, die alle drei, wenn auch von verschiedenem Standpunkte aus, für sie eintreten.

Die Differenzen, welche die Autoren trennen, liegen weniger in der makro- and mikroskopischen Beschreibung als in der Auffassung der Histogenese. Während von den deutschen Autoren, Philippson und Török die Geschwülste als epitheliale ansehen und von hypothetischen, epithelialen, embryonalen Keimen ableiten, treten Beneke und Jarisch für die bindegewebige Natur derselben ein. Beneke lässt die Zellwucherung von den Lymphgefässen, Jarisch von den Gefässendothelien ausgehen.

Bei so differenter Auffassung könnte ein Zweifel entstehen, ob der von mir beschriebene, nach meiner Ansicht ganz eindeutige Fall auch zu dieser Gruppe gehört. Indessen wird, wer sich die Mühe nimmt, die Zeichnungen von Beneke und Jarisch mit den meinen zu vergleichen, und die Uebereinstimmung der makroskopischen und grösstentheils mikroskopischen Beschreibung berücksichtigt, keinen Zweifel hegen können.

Ich will mich darauf beschränken, die Differenzen zwischen den Fällen Beneke, Jarisch und mir zu besprechen.

Jarisch wird veranlasst, den Namen Hämangioendotheliom zu wählen, weil einzelne Zellstränge und Colloidcysten offenbar mit Gefässen im Zusammenhang stehen. Ich kann diesen Ausnahmebefund bestätigen. Den Zellstrang h, der 4 seitliche Auswüchse zeigt, halte ich für ein Gefäss, ebenso mit Bestimmtheit die deutlich doppelreihige Zellenfolge $\mathrm{i}$ mit seitlichem cystischem Auswuchs nach Lage und Aussehen für eine Papillarcapillare. Hieraus ergiebt sich indessen noch nicht die Nothwendigkeit,

1) Die Beziebungen des Colloid-Milium (E. Wagner), der colloiden Degeneration der Haut (Besnier) und des Hydradenoms (JaquetDarier). Monatschr. f. prakt. Derm. 1890. Bd. XI und Referat von Philippson über den von Lesser-Beneke (a. a. 0.) bescbriebenen Fall. Monatsh. f. prakt. Dermat. Bd. XII. S. 233.

2) a. a. 0 .

3) Ueber die capillären Lymphangiome der Haut und über die Beziehungen des Lymphangioma u. s. w. Monatsh. f. prakt. Dermat. 1892. Bd. XIV. S. 169. 
das Endothel der Gefässe für die anhängenden Zellwucherungen in Anspruch zu nehmen; diese können von den die Gefässe stets begleitenden adventitiellen Bindegewebszellen ausgehen, wie mir das bei Besichtigung mit starker Vergrösserung auch als wahrscheinlich erscheint. Auf diesen Ausnahmebefund hin nun aber auch die Genese der zahlreichen Zellwucherungen in der gefässarmen Cutis propria stützen zu wollen, scheint mir um so bedenklicher, als gerade die oberflächlichen gefässreichen Cutisschichten, die Cutis vascula Beneke und mir von Geschwulstelementen fast vollkommen frei sind, während der eigentliche Sitz der Geschwulst die Cutis propria ist, welche an sich gefüsslos nur die Gefässe einschliesst, die vom subcutanen Gewebe zur Cutis vasculosa ziehen.

Beneke hat die alte Hebra-Kaposi'sche Bezeichnung Lymphangiom beibehalten. Er hält es für conventionell, ob man eine Geschwulst, die von den die Lymphgefässe auskleidenden Endothelien ausgeht, als Endotheliom oder Lymphangiom (Lymphangioendotheliom) bezeichnen soll. Ich stimme mit ihm darin überein, dass den letzten Grund für die jeweilige Bezeichnung die Frage bildet: wo hören die Lymphgefässe auf und wo fangen die Lymph- oder Gewebsspalten an?

In der Haut bestehen diesbezüglich folgende Verhältnisse. Nach den Untersuchungen von Teichmann ${ }^{1}$ ), Neumann ${ }^{2}$ ) und mir ${ }^{3}$ ) liegt an der unteren und oberen Grenze der Catis propria ein lymphatisches, capillares Netzsystem, während die Cutis selbst keine Lymphcapillaren aufweist, ausser den Verbindungskanälen zwischen unterem und oberem Lympheapillarnetz. Das subpapillare, uns vorwiegend interessirende Lymphgefässnetz lässt sich leicht durch oberflächliche Injection mit Berlinerblau injiciren. Untersucht man ein derartig injicirtes Hautstück mikroskopisch nach Tingirung der Kerne, so fiudet man an den injicirten Kanälen wohl hie und da einen Zellkern anliegen; ein zusammenhängendes Endothel ist indessen nicht zu constatiren, vielmehr will es erscheinen, dass die Infectionsmasse vielfach

1) Das Saugadersystem vom anatomischen Standpunkte. 1861.

2) Die Lympbgefässe der Baut. Wien 1873.

3) Lympbbabnen und Lymphcirculation der Haut. Monatshefte f. prakt. Dermat. 1891. Bd. XIII. 359. 
ganz direct an die collagenen Gewebsbündel angrenzt und dass diese im Verein mit elastischen Fasern die Wandung der Capillaren bilden. Diese besässen hiernach keine eigene zusammenhängende Endothelauskleidung, sondern lägen - wenigstens zum Theil - nackt, gleich den Bindegewebs- oder Lymphspalten, in der Grundsubstanz des Bindegewebes. Diese Auffassung erhält durch folgende Versuche eine Stütze. Hat man das oberflächliche Lymphnetz durch eine vorsichtige Injection mit Berlinerblau unter geringem Drucke eben injicirt, so erscheinen die Contouren der Capillaren zum grössten Theil rund, glatt, so dass man sie als geschlossene Kanäle aufzufassen versucht ist. Erhöht man indessen den Druck über das nothwendige Maass nur um Geringes, so verschwindet der glatte Contour der Capillaren und an ihre Stelle treten zahlreiche grössere und kleinere Zacken und Ausläufer: Aus den scheinbar geschlossenen Kanälen ist nach allen Richtungen Injectionsmasse ausgetreten. Noch prägnanter wird der Versuch, wenn man dünnflüssigeres Injectionsmaterial verwendet (Asphalt-Terpenthin). Hier fliesst aus den bei der Injection anfänglich anschiessenden Capillaren auch unter geringstem Drucke die Masse in die Gewebsspalten nach allen Richtungen hin, so dass der Verlauf der Capillaren für das Auge fast verschwindet.

Aus diesen Injectionsversuchen geht meiner Meinung nach mit Sicherheit hervor, was ja auch die mikroskopische Untersuchung zeigte, dass die als Capillaren bezeichneten Lymphkanäle keine geschlossene Wandung besitzen, sondern nach allen Richtungen auf das Ausgiebigste mit den Bindegewebsspalten communiciren. Wir können sie uns daher wohl am besten als erweiterte und besonders grosse Gewebsspalten vorstellen, deren Wandung hie und da eine Bindegewebszelle auskleidet.

Solche erweiterten Lymphspalten oder -Capillaren besitat nun die Cutis propria nicht. Es gelingt hier überhaupt eine gute Injection der Gewebsspalten nur mit Asphaltlösungen. An einer derartigen Injection erkennt man, dass die Cutis einen ungeheuren Reichthum an feinsten Gewebsspalten besitzt, die nackt $z$ wischen den elastischen und collagenen Fasern verlaufen und diese von allen Seiten umschliessen.

Diese Verhältnisse als richtig vorausgesetzt, können wir von 
einem Endothel der Lymphgefässe der Cutis im Gegensatz zu den festen Bindegewebszellen nicht reden und müssen daher auch die Bezeichnung unserer Geschwulst als Lymphangioma oder Lymphangioendothelioma als nicht berechtigt erachten.

Das Vorhandensein grösserer Colloidcysten in den oberen Cutislagen, das Beneke mit Veranlassung war, die Geschwulst von dem vermutheten Endothel der dort befindlichen Lymphcapillaren abzuleiten, führe ich auf den geringen Gewebsdruck zurück, der auf den oberflächlichen, nur von der Parenchymhaut bedeckten Zellwucherungen lastet.

Ich schlage in Anlehnung an die Kaposi'sche Bezeichnung den Namen Endothelioma tuberosum multiplex vor und möchte das Adjectivum colloides hinzufügen, da die colloide Entartung der Geschwulst das cystische Gepräge verleiht.

II. A. Z., Landwirth, 41 Jahre. Angeblich seit vielen Jahren besteht unter dem rechten Auge ein fast erbsengrosser gelblicher transparenter Tumor, der sich kugelartig über die Haut erhebt. Oberfläche glatt; feste Cohärenz mit der Cutis. Die mikroskopische Untersuchung des excidirten Tumors ergiebt das Vorhandensein von 5 grossen, mit Colloid gefüllten Cysten, deren Wandungen verschiedentlich mit ein bis mebreren Zelllagen ausgekleidet sind. Die Zellen theils abgeplattet, endothelartig, theils cubisch bis cylinderförmig, epithelartig. An einzelnen Stellen liegen die Colloidmassen nackt dem Bindegewebe an, dem Anscheine nach in Folge von Atrophie der auskleidenden Zellen durch den Druck des Colloids. Ceber die dicht auf einander gepresst liegenden Cysten zieht die Parenchymhaut (Epidermis mit Cutis vasculosa) atrophisch obne Papillenbildung aber sonst unverändert hinweg. In der Umgebung der grossen Cysten ist die Cutis in ganz gleicher Weise wie im vorigen Falle verändert, so dass die Genese der Cysten dadurch erklärt wird: Endotbelioma tuberosum colloides cysticum.

\section{Erklärung der Abbildungen.}

Taf. IX. Fig. 1-2.

Fig. 1. Querschnitt durch den Tumor, schwache Vergrösserung. a, b, c Zell. reihen. d, e, f. g Zellstränge. h, i Blutgefüsse mit eystischen Anbängen. $\mathrm{k}$ grössere Cyste mit theilweise ausgefallenen Colloidmassen.

Fig. 2. Zellstrang mit cystischer Erweiterung a. Bei b, c, d Verbindung des Zellstranges mit Bindegewebszellen der Cutis. e Zellreihe. 